EPJ Web of Conferences 29, 00035 (2012)

DOI: $10.1051 /$ epjconf/20122900035

C) Owned by the authors, published by EDP Sciences, 2012

\title{
Effects of the random field on the magnetic behavior of a nanoparticle with core/shell morphology
}

\author{
A. Zaim ${ }^{\text {a }}$, M. Kerouad and M. Boughrara \\ Laboratoire Physique des Matériaux et Modélisation des Systèmes (LP2MS), Unité Associée au \\ CNRST-URAC: 08, Faculty of Sciences, University Moulay Ismail, B.P. 11201 Zitoune, Meknes, \\ Morocco.
}

\begin{abstract}
We have used the effective field theory based on probability distribution method to investigate the hysteresis behavior of a magnetic nanoparticle with core/shell morphology in a random field. The hysteresis curves are obtained for different values of the random field and the temperature. We find a number of characteristic behaviors, such as the existence of double hysteresis loops for appropriate values of the system parameters. The remanent magnetization and the coercive field as a function of the temperature are examined.
\end{abstract}

\section{Introduction}

Magnetic nanoparticles have attracted a strong interest because of their outstanding properties, which is a result of their small size, and where both surface effects become dominant and quantum size effects occur. Magnetic nanoparticles have high potential for applications in diverse areas of highdensity magnetic storage media [1, 2], magnetic resonance imaging [3], non-linear optics [4], ferrofluids [5], environmental remediation [6], catalysis [7] and biomedicine [8]. Theoretically, the magnetic properties of nanoparticles have been widely investigated by the use of various techniques, including the mean field model [9], effective-field theory [10-12], Monte Carlo simulations [13-16], Green's function technique [17-18] and Bethe Peiris approximation [19]. Some studies show that we can find in these systems a very rich critical behavior and many interesting phenomena. Zaim et al. [16] have discussed the magnetic behaviour of a ferrimagnetic core/shell nanoparticle. They have found that the system can exhibit one or even two compensation temperatures. Experimentally, the magnetic properties of magnetic nanoparticles were characterized by Mössbauer spectra, magnetization curve, ferromagnetic resonance [20], and EPR [21]. The forth is the applications of the nanoparticles and their assembly in various fields.

In particular, we call attention to the work of Kaneyoshi, [22] where he investigated the magnetization and the phase diagram of a nanoparticle described by the transverse Ising model within the two theoretical frameworks, namely the standard mean-field theory and the effective-field theory. However, the study the effects of the random magnetic field on the hysteresis behaviours of a nanoparticle with core/shell morphology has not been investigated. Therefore, the purpose of this study is to investigate the hysteresis behaviors of an Ising nanoparticle, using an effective field

\footnotetext{
a e-mail : ah_zaim@yahoo.fr
} 
theory based on a probability distribution method. We also study the temperature dependence of the coercivities and remanent magnetizations.

The paper is arranged as follows: In Section 2, we give the model and the formalism. In Section 3 we present the results and discussions, while section 4 is devoted to a brief conclusion.

\section{Model and formalism}

We consider an Ising nanoparticle model consists of two dimensional arrangement of spins, disposed in concentric hexagonal rings [22]. As shown in fig .1, the particle is composed of the surface shell and the core in which the core surrounded by the surface shell. Here, the model is taken into account the random magnetic field and the external magnetic field, which is quite different from that of Ref. [22]. The Hamiltonian of the model is given by

$$
\mathrm{H}=-\sum_{\langle i j\rangle} J_{i j} \sigma_{i} \sigma_{j}-\sum_{i} h_{0 i} \sigma_{i}-h \sum_{i} \sigma_{i}
$$

Where $\sigma_{\mathrm{i}}= \pm 1$ is the usual Ising variable and $\mathrm{J}_{\mathrm{ij}}$ is the exchange interaction. $\mathrm{J}_{\mathrm{ij}}$ takes the value $\mathrm{J}_{\mathrm{s}}$ if both pseudo-spins belong to the surface ring and the value $J_{c}$ otherwise. $h$ is an external magnetic field, $\mathrm{h}_{0 \mathrm{i}}$ is the random magnetic field acting on $\sigma_{\mathrm{i}}$, distributed according to a bimodal distribution:

$$
p\left(h_{0 i}\right)=\frac{1}{2}\left[\delta\left(h_{0 i}-h_{0}\right)+\delta\left(h_{0 i}+h_{0}\right)\right]
$$

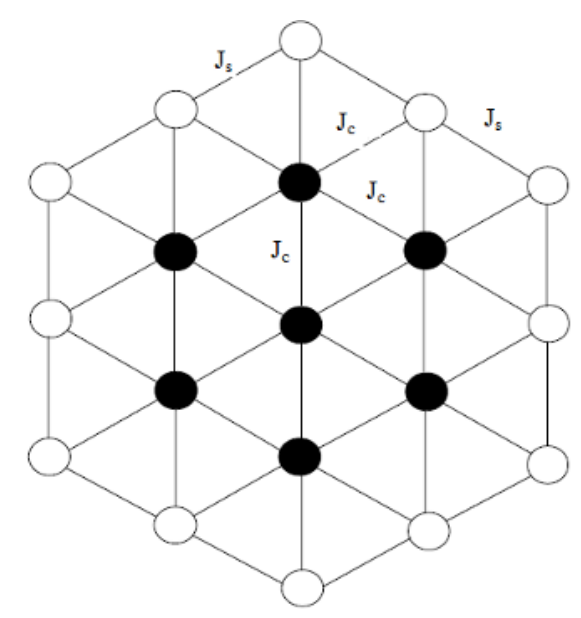

Fig. 1. Schematic representation of a nanoparticle. Open circles indicate magnetic atoms at the surface shell and solid circles are magnetic atoms constituting the core.

Two longitudinal magnetizations on the shell surface, namely $\mathrm{m}_{\mathrm{s} 1}$ and $\mathrm{m}_{\mathrm{s} 2}$, and two longitudinal magnetizations on the core, namely $\mathrm{m}_{\mathrm{c} 1}$ and $\mathrm{m}_{\mathrm{c} 2}$, within the framework of the effective field theory with a probability distribution technique, can be obtained as,

$$
\begin{aligned}
m_{s 1}= & 2^{-3} \sum_{\mu=0}^{2} \sum_{\mu 1=0}^{1} C_{\mu}^{2} C_{\mu 1}^{1}\left(1-2 m_{s 2}\right)^{\mu}\left(1+2 m_{s 2}\right)^{2-\mu}\left(1-2 m_{c 2}\right)^{\mu 1} \\
& \times\left(1+2 m_{c 2}\right)^{1-\mu 1}\left\{\frac{f\left(X_{s 1}, h, h_{0}\right)+f\left(X_{s 1}, h,-h_{0}\right)}{2}\right\} \\
m_{s 2}= & 2^{-4} \sum_{\mu=0}^{2} \sum_{\mu 1=0}^{2} C_{\mu}^{2} C_{\mu 1}^{2}\left(1-2 m_{s 1}\right)^{\mu}\left(1+2 m_{s 1}\right)^{2-\mu}\left(1-2 m_{c 2}\right)^{\mu 1} \\
& \times\left(1+2 m_{c 2}\right)^{1-\mu 1}\left\{\frac{f\left(X_{s 2}, h, h_{0}\right)+f\left(X_{s 2}, h,-h_{0}\right)}{2}\right\}
\end{aligned}
$$




$$
\begin{gathered}
m_{c 1}=2^{-6} \sum_{\mu=0}^{6} C_{\mu}^{6}\left(1-2 m_{c 2}\right)^{\mu}\left(1+2 m_{s 2}\right)^{6-\mu}\left\{\frac{f\left(X_{c 1}, h, h_{0}\right)+f\left(X_{c 1}, h,-h_{0}\right)}{2}\right\} \\
m_{c 2}=2^{-6} \sum_{\mu=0}^{1} \sum_{\mu 1=0}^{2} \sum_{\mu 2=0}^{1} \sum_{\mu 3=0}^{2} C_{\mu}^{1} C_{\mu 1}^{2} C_{\mu 2}^{1} C_{\mu 3}^{2}\left(1-2 m_{c 1}\right)^{\mu}\left(1+2 m_{c 1}\right)^{1-\mu}\left(1-2 m_{c 2}\right)^{\mu 1}\left(1+2 m_{c 2}\right)^{2-\mu 1} \\
\times\left(1-2 m_{s 1}\right)^{\mu 2}\left(1+2 m_{s 1}\right)^{1-\mu 2}\left(1-2 m_{s 2}\right)^{\mu 3}\left(1+2 m_{s 2}\right)^{2-\mu 3}\left\{\frac{f\left(X_{c 2}, h, h_{0}\right)+f\left(X_{c 2}, h,-h_{0}\right)}{2}\right\}
\end{gathered}
$$

where

$$
\begin{aligned}
X_{s 1}= & J_{s}(2-2 \mu)+J_{c}(1-2 \mu 1), \\
X_{s 2}= & J_{s}(2-2 \mu)+J_{c}(2-2 \mu 1), \\
& X_{c 1}=J_{c}(6-2 \mu),
\end{aligned}
$$

and

$$
X_{c 2}=J_{c}(1-2 \mu)+J_{c}(2-2 \mu 1)+J_{c}(1-2 \mu 2)+J_{c}(2-2 \mu 3) .
$$

The function is given by

$$
f\left(X, h, h_{0}\right)=\tanh \left(\beta\left(X-h-h_{0}\right)\right)
$$

With $\beta=1 / \mathrm{k}_{\mathrm{B}} \mathrm{T}, \mathrm{T}$ is the absolute temperature and $\mathrm{k}_{\mathrm{B}}$ is the Boltzmann factor. $C_{k}^{l}$ denote the binomial coefficient, $C_{k}^{l}=l ! / k !(l-k) !$.

Furthermore, let us define the longitudinal magnetizations per site of the shell surface $M_{s}$, the core $\mathrm{M}_{\mathrm{c}}$, and the total longitudinal magnetization of per site $\mathrm{M}_{\mathrm{T}}$ as follows:

$$
\begin{gathered}
M_{s}=\frac{1}{2}\left(m_{s 1}+m_{s 2}\right), \\
M_{c}=\frac{1}{7}\left(6 m_{c 2}+m_{c 1}\right), \\
M_{T}=\frac{1}{19}\left(6\left(m_{s 2}+m_{s 2}\right)+6 m_{c 2}+m_{c 1}\right) .
\end{gathered}
$$

The coercive field $h_{c}$ is defined by

$$
h_{c}=\frac{h_{\text {right }}-h_{\text {left }}}{2}
$$

with $\mathrm{h}_{\text {right }}$ and $\mathrm{h}_{\text {left }}$ being the points where the loop intersects the field axis.

The remanent magnetization $\mathrm{M}_{\mathrm{r}}$ is defined by

$$
M_{r}=\frac{M_{1}-M_{2}}{2}
$$

with $\mathrm{M}_{1}$ and $\mathrm{M}_{2}$ are the values of positive and negative magnetization at a zero external field.

\section{Results and discussions}

The total magnetization per spin $\mathrm{M}_{\mathrm{T}}$ as a function of the random magnetic field $\mathrm{h}_{0}$ for $\mathrm{J}_{\mathrm{S}} / \mathrm{J}_{\mathrm{c}}=1$, $\mathrm{T} / \mathrm{J}_{\mathrm{c}}=0.25$ and the absence of the external magnetic field $\mathrm{h}=0$, is shown in Fig. 2 . We see that the first-order transition from the ferromagnetic $\left(M_{T} \neq 0\right)$ to the paramagnetic $\left(M_{T}=0\right)$ state takes at $\mathrm{h}_{0 \mathrm{c}}=2.303$. This type of behaviour of first-order transition has not observed in Ref. [22] where the model is studied without the random magnetic field. 


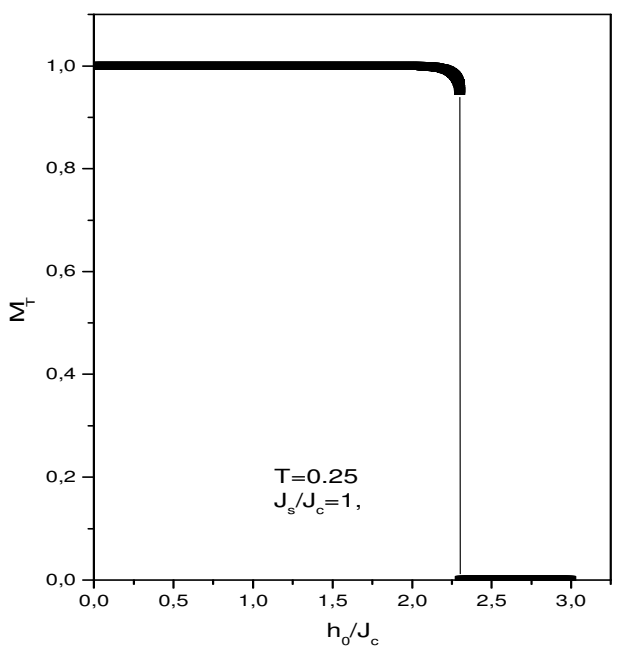

Fig. 2. The total magnetization $M_{T}$ as a function of the random magnetic field $h_{0}$ for $J_{s} / J_{c}=1, T / J_{c}=0.25$ and $\mathrm{h}=0$.

In order to investigated the influence of the random magnetic field $h_{0}$ on the hysteresis behavior of a ferromagnetic nanoparticle, a series of hysteresis loops for two regimes $\left(h_{0}<h_{0 c}\right.$ and $\left.h_{0} \geq h_{0 c}\right)$ is plotted in Fig. 3 with the fixed parameters, $\mathrm{J}_{\mathrm{s}} / \mathrm{J}_{\mathrm{c}}=1$ and $\mathrm{T} / \mathrm{J}_{\mathrm{c}}=0.25$. From Fig. $3 \mathrm{a}\left(\mathrm{h}_{0}<\mathrm{h}_{0 \mathrm{c}}\right)$, we can see that only a normal hysteresis loop and that with increasing $\mathrm{h}_{0}$, the zone of the loop decreases. We also see that two steps appear when the random magnetic field $h_{0}$ approach to its critical value $h_{0 c}=2.303$. For $\mathrm{h}_{0} \geq \mathrm{h}_{0 \mathrm{c}}$ (Fig. 3b), it is found that the shapes of the hysteresis loops changed from ferromagnetic hysteresis loop to double hysteresis loops and that when $\mathrm{h}_{0}$ increases, the double hysteresis loops stretch further out horizontally.
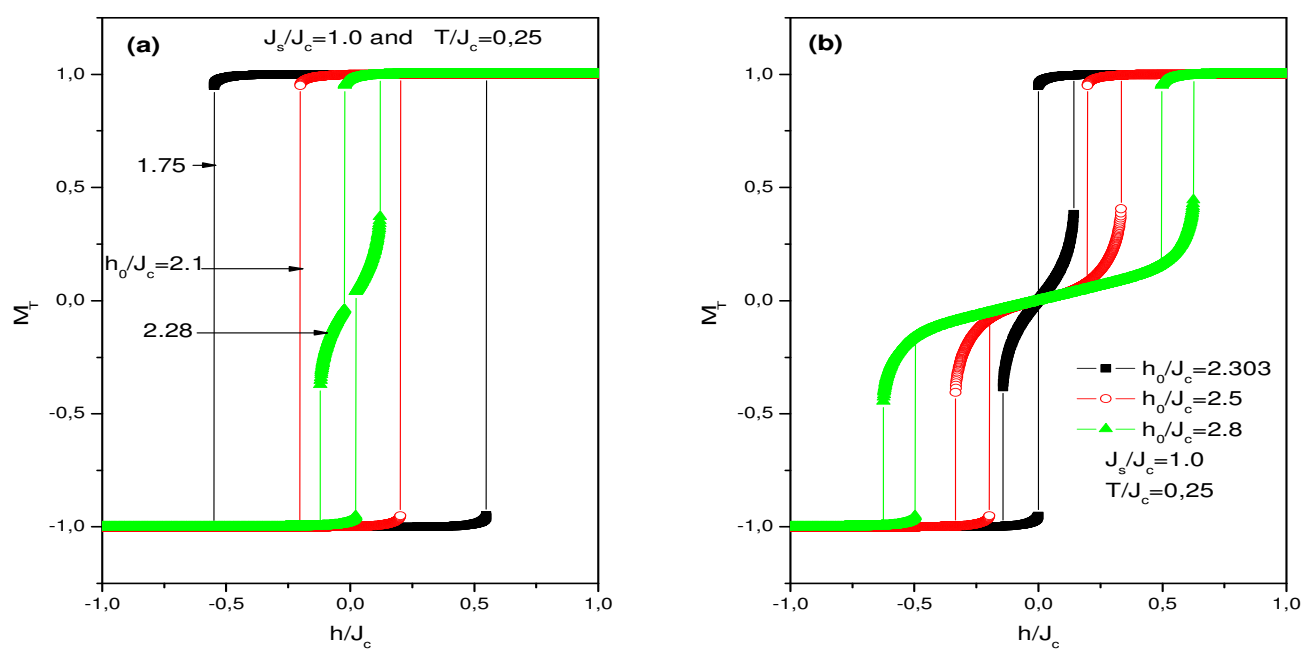

Fig. 3. The hysteresis loops for two regimes (a) $h_{0}<h_{0 c}$ and (b) $h_{0} \geq h_{0 c}$ with the fixed parameters, $\mathrm{T} / \mathrm{J}_{\mathrm{c}}=0.25, \mathrm{~J}_{\mathrm{s}} / \mathrm{J}_{\mathrm{c}}=1$.

The dependence of the hysteresis loops on the temperature $T$ for $J_{s} / J_{c}=1$ and for $h_{0} / J_{c}=2.05$ is shown in fig. 4 , it is seen that with increasing the temperature, the hysteresis loop is more compact 
and lower; and the coercive field decreases. For $T / J_{c}>T_{c} / J_{c}$ the hysteresis loop disappears $\left(T_{c}=2.6\right)$. The coercive field and its slope vanished at the curie temperature. We can see that when the temperature increases, the remanent magnetization decreases from its saturation value at low temperature region and vanishes at the critical temperature.
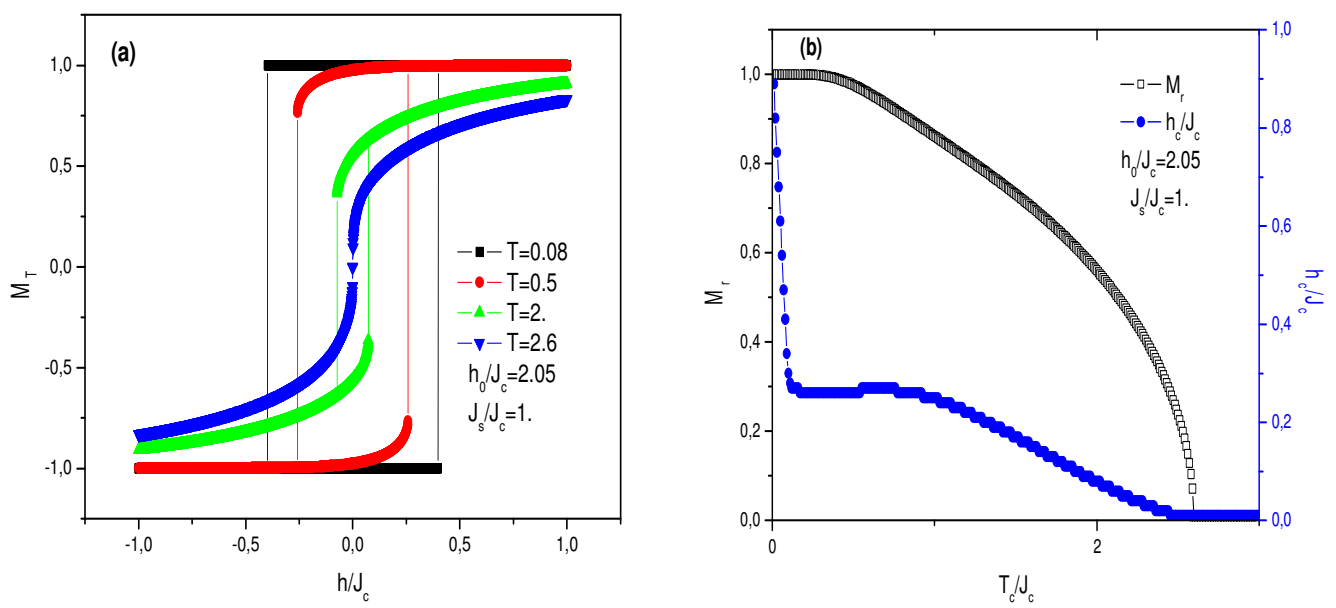

Fig. 4. a) The hysteresis loops for different values of the temperature with the fixed parameters, $\mathrm{T} / \mathrm{J}_{\mathrm{c}}=0.25, \mathrm{~J}_{\mathrm{s}} / \mathrm{J}_{\mathrm{c}}=1$. b) The dependence of the coercive field $\mathrm{h}_{\mathrm{c}} / \mathrm{J}_{\mathrm{c}}$ and the remanent magnetization $\mathrm{M}_{\mathrm{r}}$ as a function of the temperature $T / J_{c}$.

\section{Conclusion}

In summary, we have studied the hysteresis behaviour of the ferromagnetic nanoparticle on an Ising model, where we have taken the coupling constants $\mathrm{J}_{\mathrm{c}}$ and $\mathrm{J}_{\mathrm{s}}$ for the core and the surface shell respectively. Using an effective field theory based on a probability distribution method we have discussed the influence of the random field on the magnetic properties of a nanoparticle with core/shell morphology. It is shown that the shapes of the hysteresis loops changed from ferromagnetic hysteresis loop to double hysteresis loops and that when $\mathrm{h}_{0}$ increases, the double hysteresis loops stretch further out horizontally. It is also shown that, the coercive field and the remanent magnetization decreases with increasing temperature.

\section{Acknowledgement}

This work has been supported by URAC: 08, and the project No: A/030519/10 financed by A.E.C.I.

\section{References}

1. R. H. Kodama, A. E Berkowitz, E. J McNiff, Jr, S Foner, 'Surface spin disorder in NiFe2O4 nanoparticles' Phys. Rev. Lett. 77, 394 (1996)

2. T. Hayashi, S. Hirono, M. Tomita and S. Umemura, Nature 381, 772 (1996)

3. J. Kim, S. Park, JE. Lee, SM. Jin, JH. Lee, IS. Lee, I. Yang, JS. Kim, SK. Kim, MH. Cho and T. Hyeon, Angew. Chem. Int. Ed. 45, 7754, 2006

4. S. Nie and S. R. Emory, Science 275, 1102, 1997

5. R. E. Rosensweig, Ferrohydrodynamics (New York: Dover), 1997 
6. D. W. Elliott, W.-X. Zhang, Environ. Sci. Technol. 35, 4922 (2001)

7. A.-H. Lu, W. Schmidt, N. Matoussevitch, H. Bönnemann, B. Spliethoff, B. Tesche, E. Bill, W. Kiefer, F. Schüth, "Nanoengineering of a Magnetically Separable Hydrogenation Catalyst". Angewandte Chemie International Edition 43 (33) 4303, 2004.

8. A. P. Y. Wong and M. H. W. Chan, Phys. Rev. Lett. 65, 2567 (1990).

9. F. Michael, C. Gonzalez, V. Mujica, M. Marquez and M. A. Ratner Phys. Rev. B 76, 224409 (2007).

10. T. Kaneyoshi, J. Magn. Magn. Mater. 322, 3410 (2010).

11. T. Kaneyoshi, J. Magn. Magn. Mater. 322, 3414 (2010.

12. M. Keskin, N. Sarli and B. Deviren, Solid State Communications. 151, 1025 (2011).

13. A. Zaim, M. Kerouad, Y. El Amraoui, J. Magn. Magn. Mater. 321, 1077 (2009).

14. O. Iglesias, X. Batlle and A. Labarta, Phys. Rev. B 72, 212401 (2005).

15. M. Vasilakaki and K. N. Trohidou, Phys. Rev. B 79, 144402 (2009).

16. A. Zaim, M. Kerouad, Physica A 389, 3435 (2010).

17. J. M. Wesselinowa, J. Magn. Magn. Mater. 322, 234 (2010).

18. J. M. Wesselinowa, I. Apostolova, Journal of Applied Physics 104, 084108 (2008).

19. L. G. C. Rego and W. Figueiredo, Phys. Rev. B 64, 144424 (2001).

20. E. L. Bizdoaca, M. Spasova, M. Farle, M. Hilgendorff, F. J. Caruso, Magn. Magn. Mater. 240, 44 (2002).

21. M. Droz, A. Maritan, and A. L. Stella, Phys. Lett. A 92, 287 (1982).

22. T. Kaneyoshi, Phys. Stat. Sol. (b) 242, 2938 (2005). 